\title{
Research of Power Distribution System Dynamic Simulation Based on Grid's Harmonic Current
}

\author{
Wei QIN ${ }^{\mathrm{a}, 1}$, Tiansong GU ${ }^{\mathrm{b}}$ and Hongliang $\mathrm{LI}^{\mathrm{b}}$ \\ ${ }^{a}$ Wuhan Nari Limited Library Company of State Grid Electric Power Research \\ Institute, China \\ ${ }^{\mathrm{b}}$ State Grid Jibei Electric Power Company Limited, China
}

\begin{abstract}
Harmonic current in power grid will cause extra power consumption of electrical equipment and affect the stable operation of power grid. Based on the dynamic model experiment system of $10 \mathrm{kV}$ distribution network, a working platform for parallel operation of charging pile was built, which studied the harmonic current components and analyzed the simulation waveform. The results show that with the increase of number of charging piles, the content of harmonic current will decrease correspondingly, the main components are low frequency odd harmonics; When 16 charging piles are connected in parallel, three harmonics are the main components; the waveform of the simulation calculation curve and the actual measurement curve are basically consistent, and the current amplitude of each frequency harmonic wave is basically the same, so it is proved that the dynamic model test platform is reasonable and feasible to test grid's harmonic current. The dynamic model can provide technical support for harmonic suppression research.
\end{abstract}

Keywords. Charging pile, LCL, harmonic current, dynamic simulation, artificial load.

\section{Introduction}

With the introduction of document "The 13th Five-Year Plan for the Development of National Strategic Emerging Industries", new energy vehicles will occupy the main market of road traffic. Charing pile(CP), as the power supply device of new energy vehicles, is everywhere in each station. Due to the existence of power electronic devices in CP, capacitance and inductance will cause the instability of the power grid. Therefore, scholars at home and abroad have carried out studies on the suppression of parallel resonance characteristics, and have proposed a virtual resistance damping method based on capacitance and voltage feedback, so as to realize the stable operation of the system [1]. The dynamic model experiment system is essentially the operation platform of the distribution network equipment that simulates full voltage and full current. According to the principle of similarity and the requirements of the equipment, an experiment platform can be established to simulate the real scene. Reference [2] uses equipment such as fault indicator and traveling wave tester to carry out static detection, thus improving the overall performance. In this reference [3], the overhead and cable mixed line distribution

\footnotetext{
${ }^{1}$ Corresponding Author; E-mail: qwqc1012@qq.com.
} 
network for new energy system access is built, which can carry out grounding fault analysis of different types and related tests of other automatic equipment.

At present, CP test research is limited to simulation analysis or power network simplification research, and has not carried out the construction of the whole distribution network. Its performance test has a certain gap with the practical operation results, which is not convincing. Dynamic simulation experiment system has studied the electrical characteristics of different power equipment, such as capacitors, switches, etc., but lack of CP test research. Therefore, CP is taken as the test object and a dynamic model experiment platform is built to carry out the harmonic current test of $\mathrm{CP}$.

\section{CP parallel model}

CP is equipped with LCL active power filter, which adopts modern power electronics technology to automatically track harmonic current generated in the power grid. CP can compensate or cancel harmonic current to ensure the stability and reliability of power grid voltage. The single-phase CP system structure is shown in Fig.1.

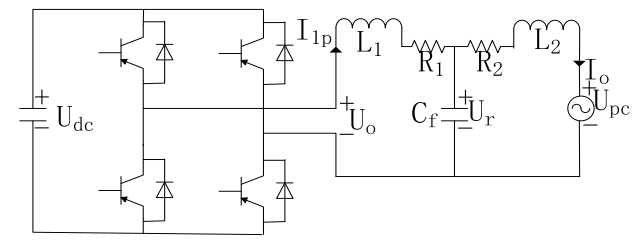

Figure 1. Structural diagram of single-phase CP system

In Fig. 1, $U_{d c}$ is the DC input voltage; $I_{l p}$ is the closed-loop current control value at the converter side; $U_{r}$ is the capacitor voltage; $U_{o}$ is the output voltage; $I_{o}$ is the output current of the converter network side, and $U_{p c}$ is the voltage of the converter access point.

\subsection{Single-phase CP closed-loop model}

Single-phase CP is controlled by a double closed-loop network of closed-loop current $I_{l p}$ and capacitor voltage $U_{r}$, so as to suppress the resonance phenomenon of the system. The established single-phase CP current and voltage transfer function is shown in Fig.2.

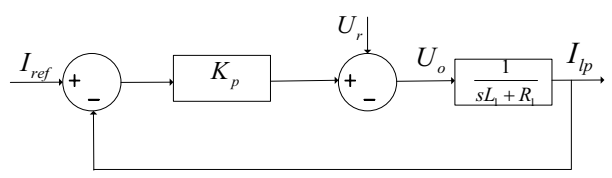

Figure 2. Current and voltage transfer function of single $\mathrm{CP}$

The output voltage can be calculated as:

$$
U_{o}(s)=U_{r}(s)+K_{p}(s)\left[I_{r e f}(s)-I_{l p}(s)\right]
$$


The transfer function of important parameters in the system structure is given by:

$$
\begin{aligned}
& I_{l p}(s)=H_{l 1}(s) U_{o}(s)+H_{l 2}(s) U_{i}(s) \\
& I_{o}(s)=H_{o 1}(s) U_{o}(s)+H_{o 2}(s) U_{i}(s) \\
& U_{r}(s)=H_{r 1}(s) U_{o}(s)+H_{r 2}(s) U_{i}(s)
\end{aligned}
$$

Among them:

$$
\begin{aligned}
& H_{l l}(s)=\frac{L_{2} C_{f} s^{2}+R_{2} C_{f} s+1}{L_{1} L_{2} C_{1} s^{3}+\left(R_{2} L_{1}+R_{1} L_{2}\right) C_{f} s^{2}+\left(L_{1}+L_{2}+C_{f} R_{1} R_{2}\right) s+R_{1}+R_{2}} \\
& H_{l 2}(s)=\frac{-1}{L_{1} L_{2} C_{s} s^{3}+\left(R_{2} L_{1}+R_{1} L_{2}\right) C_{f} s^{2}+\left(L_{1}+L_{2}+C_{f} R_{1} R_{2}\right) s+R_{1}+R_{2}} \\
& H_{o 1}(s)=\frac{1}{L_{L} L_{2} C_{1} s^{3}+\left(R_{2} L_{1}+R_{1} L_{2}\right) C_{f} s^{2}+\left(L_{1}+L_{2}+C_{f} R_{1} R_{2}\right) s+R_{1}+R_{2}} \\
& H_{o 2}(s)=\frac{-\left(L_{2} C_{f} s^{2}+R_{2} C_{f} s+1\right)}{L_{1} L_{2} C_{1} s^{3}+\left(R_{2} L_{1}+R_{1} L_{2}\right) C_{s} s^{2}+\left(L_{1}+L_{2}+C_{f} R_{1} R_{2}\right) s+R_{1}+R_{2}} \\
& H_{r 11}(s)=\frac{L_{2} s+R_{2}}{L_{1} L_{2} C_{s} s^{3}+\left(R_{2} L_{1}+R_{1} L_{2}\right) C_{f} s^{2}+\left(L_{1}+L_{2}+C_{f} R_{1} R_{2}\right) s+R_{1}+R_{2}} \\
& H_{r 2}(s)=\frac{L_{1} s+R_{1}}{L_{1} L_{2} C_{1} s^{3}+\left(R_{2} L_{1}+R_{1} L_{2}\right) C_{f} s^{2}+\left(L_{1}+L_{2}+C_{f} R_{1} R_{2}\right) s+R_{1}+R_{2}}
\end{aligned}
$$

The closed-loop model of a single CP is as follows:

$$
I_{o}(s)=G_{T}(s) I_{r e f}(s)+Y_{e q}(s) U_{p c}(s)
$$

Where the current coefficient and equivalent admittance can be respectively given by:

$$
\begin{gathered}
G_{T}(s)=\frac{K_{p}(s) H_{o 1}(s)}{1-H_{r 1}(s)+K_{p}(s) H_{l 1}(s)} \\
Z_{e q}(s)=\frac{K_{p}(s) H_{l 2}(s) H_{o 1}(s)-H_{o 1}(s) H_{r 2}(s)}{1-H_{r 1}(s)+K_{p}(s) H_{l 1}(s)}
\end{gathered}
$$

According to NORTON theorem, CP internal circuit can be equivalent to parallel current and admittance, as shown in Fig. 3.

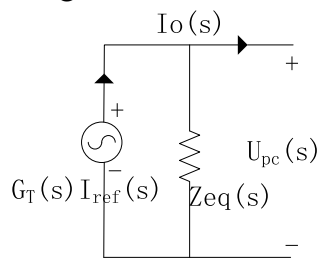

Figure 3. Norton equivalent model of $\mathrm{CP}$ circuit 


\subsection{Multi-CP parallel model}

With the development of smart power grid and the construction of smart community, CP is more and more widely used. Its connection mode is generally in parallel mode. Fig. 4 is a topology diagram of $7 \mathrm{~V} \mathrm{CP}$ connected in parallel, which is composed of NORTON equivalent circuit calculated by Equations (4) and (5). Among them, $I_{t}(s)$ is the total current after $\mathrm{CP}$ parallel connection, that is, the sum of all output currents; $U_{t}(s)$, $Z_{t}(s)$ are power grid voltage and power grid line admittance respectively.

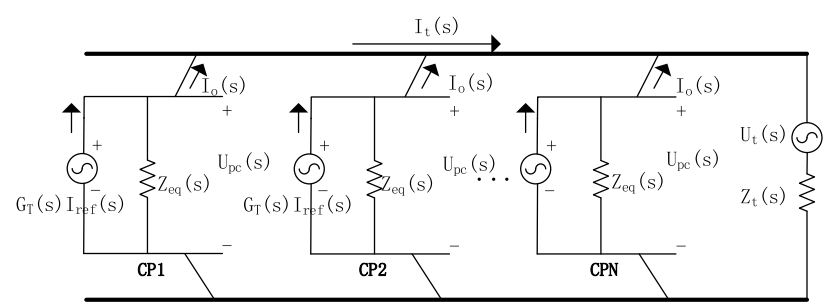

Figure 4. Network topology diagram of multi-EV chargers

To ensure the reliability of the $\mathrm{CP}$ network, if the parallel CP-general specifications and models are the same and the parameters are the same, the interface voltage can be given by:

$$
U_{p c}(s)=\frac{\sum_{i=1}^{N} G_{T}^{i}(s) I_{r e f}^{i}(s)+U_{t}(s) Z_{t}(s)}{\sum_{i=1}^{N} Z_{e q}^{i}(s)+Z_{t}(s)}
$$

Where $G_{T}^{i}(s), I_{r e f}^{i}(s), Z_{e q}^{i}(s)$ are the current coefficient, closed-loop current control value and equivalent admittance of the $\mathrm{i}_{\text {th }}$ cp respectively.

For the output current of the first $\mathrm{CP}$, then:

$$
I_{g}^{1}(s)=G_{T}^{1}(s) I_{r e f}^{1}(s)+Z_{e q}^{1}(s) U_{p c}(s)
$$

It can be obtained simultaneously:

$$
I_{g}^{1}(s)=R_{1}(s) I_{r f}^{1}(s)+\sum_{k=1}^{N} P_{1}^{k}(s) I_{r f}^{t}(s)-S_{G}^{1}(s) U_{t}(s)
$$

Among them:

$$
P_{1}^{k}(s)=\frac{-Z_{e q}^{1}(s) G_{T}^{1}(s)}{\sum_{i=1}^{N} Z_{e q}^{i}(s)+Z_{t}(s)}
$$




$$
S_{G}^{1}(s)=\frac{Z_{e q}^{1}(s) Z_{t}(s)}{\sum_{i=1}^{N} Z_{e q}^{i}(s)+Z_{t}(s)}
$$

It can be seen that $S_{G}^{1}(s) U_{t}(s)$ is the representation of the influence degree of charging pile when the grid voltage is distorted.

\section{Experimental scheme}

\subsection{Dynamic model experimental system design}

With the popularity of electric vehicles, the capacity and number of CPS have increased significantly. The capacity of DC CP has reached $250 \mathrm{KW}$, so a $10 \mathrm{KV}$ distribution network dynamic mode experiment system is built, as shown in Fig.5, which can ensure the energy supply of CP network. The whole system consists of substation, 10KV distribution line, $0.4 \mathrm{kV}$ distribution equipment, voltage and current boosting equipment, etc. $L H, R$ are used for grounding arc suppression coil and resistance to achieve nonmetallic neutral grounding, so as to limit the grounding fault current. For arc suppression coil and resistance used for grounding, non-metallic grounding of neutral point is realized, so as to limit grounding fault current; TM main transformer, capacity of 5MW, $\mathrm{L}, \mathrm{C}$ are $10 \mathrm{KV}$ reactor and $10 \mathrm{KV}$ capacitor respectively; QF is a busbar switch, which realizes different power supply forms through different switching combinations.

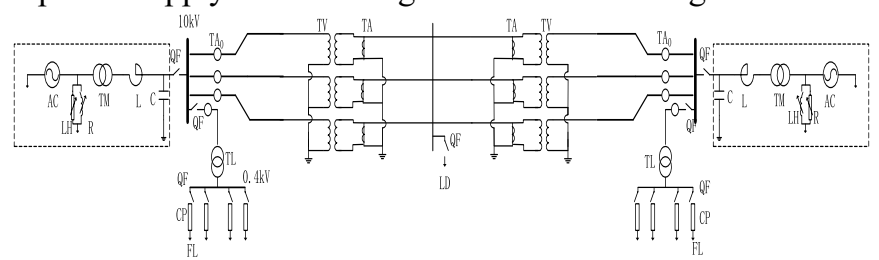

Figure 5. Topology of dynamic model for $10 \mathrm{kV}$ distribution experiment system

$\mathrm{TA}_{0}, \mathrm{TA}, \mathrm{TV}$ and LD are the loads of zero sequence current transformer, upcurrent transformer, upvoltage transformer and $10 \mathrm{kV}$ distribution network respectively. TL is the step-down transformer, realizing the transform of $10 \mathrm{kV}$ to $0.4 \mathrm{kV}$ output, and provides the input voltage for the CP network; FL is the analog load.

\subsection{Dynamic model experimental system design}

Since the neutral point of power supply adopts parallel grounding of grounding resistance and arc suppression coil, and the dynamic mode test system of distribution network operates in full working mode, the zero sequence current caused by single-phase grounding fault is very small, and the traditional current transformer cannot achieve the actual measurement accuracy, so it cannot realize the action of starting relay protection device.

As shown in Fig.6, in order to solve the accuracy problem, the primary circuit and secondary winding are wound together on the same ring core, and the secondary current value is improved through the principle of magnetic balance, so as to improve the measurement accuracy and finally meet the measurement requirements. 


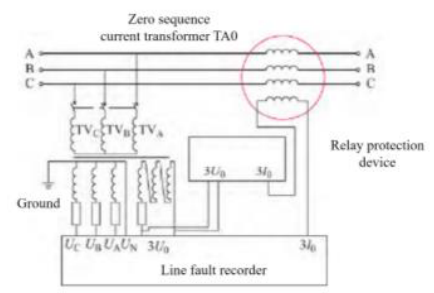

Figure 6. Structure of zero sequence current transformer

\subsection{CP network}

CP network of simulated intelligent cell is shown in Fig.7. The whole network is composed of power distribution cabinet, converter cabinet, charger, etc. Converter cabinet realizes the rectification or inverter of power grid current, so as to realize the AC and DC charging of the charger [4]. For DC charger, constant current and constant voltage are generally used for subsection charging. For example, charge the current to about 250A first and keep it stable, and then boost the voltage. When the voltage reaches a predetermined value of $1000 \mathrm{~V}$, the analog load can be charged.

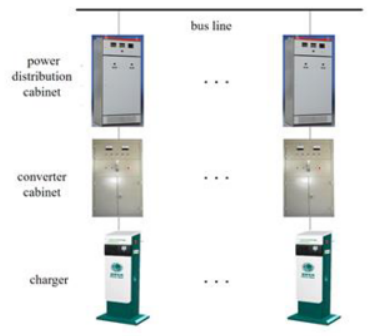

Figure 7. Network structure of $\mathrm{CP}$

\subsection{Simulated load}

The internal structure of analog load is shown in Fig.8, which is composed of battery management system (BMS) simulator, charger interface, resistance load and controller according to different interfaces. BMS simulator can not only improve battery efficiency, but also achieve battery protection. Resistance load, according to the specification and requirements of the national standard "GB/T 18487.1-2015", the nominal value is set as $1000 \Omega$ which meets between $970 \Omega$ and $1030 \Omega$.

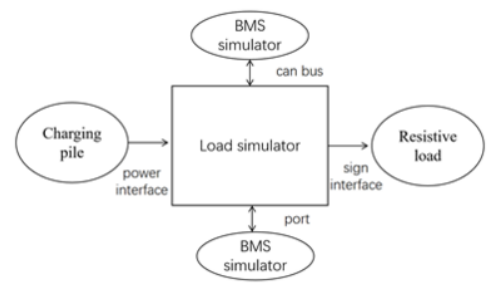

Figure 8. Internal structure of artificial load 


\section{Experimental scheme}

Due to the existence of power distribution cabinet and converter cabinet, the influence of harmonic current on power grid current cannot be ignored. In order to realize the study on harmonic current of $\mathrm{CP}$ network in intelligent residential area [5-7], a simulation experiment platform is built as shown in Fig.5. The capacity of a single CP is $250 \mathrm{~kW}$. In the experiment, the maximum value of $\mathrm{CP}$ in parallel is 16 , and the total capacity is 4 $\mathrm{MW}$, lower than the total capacity of the main transformer.

\subsection{Harmonic current measurement}

Under the normal operation of dynamic simulation experiment system, different numbers of cp are randomly connected in parallel to measure the total CP network current [8]. The summary of harmonic current data is shown in Table 1.

Table 1. Harmonic current content of different EV chargers

\begin{tabular}{cccc}
\hline number & CP number & $\begin{array}{c}\text { total harmonic } \\
\text { distortion rate } \%\end{array}$ & $\begin{array}{c}\text { the main } \\
\text { harmonic }\end{array}$ \\
\hline 1 & 1 & 4.5 & H9 \\
2 & 4 & 3.1 & H7 \\
3 & 8 & 2.9 & H5 \\
4 & 10 & 2.7 & H5 \\
5 & 16 & 1.8 & H3 \\
\hline
\end{tabular}

\subsection{Comparative analysis}

When simulating $16 \mathrm{CPs}$ in parallel, the network current value was saved and waveform was drawn, which was compared with the actual measured waveform, as shown in Fig.9.

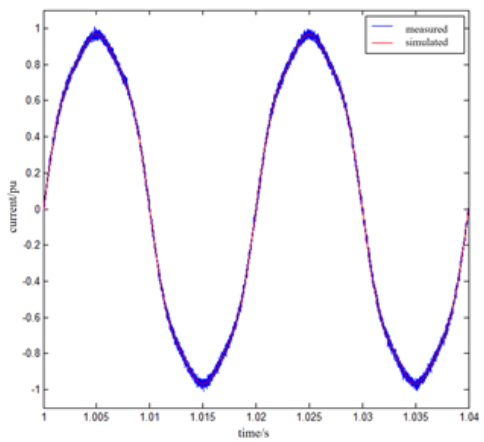

Figure 9. Comparative analysis of harmonic current in different EV chargers

It can be seen that the simulation calculation of harmonic current is basically consistent with the actual measurement curve, and there is some noise in the actual measurement curve, which may be caused by the test environment. It indicates that the dynamic mode experimental system is feasible to measure harmonic current. 


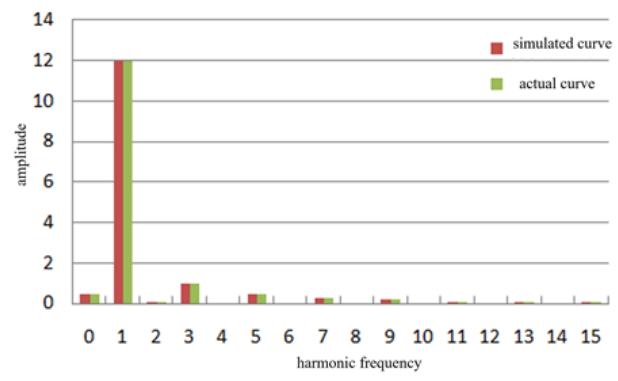

Figure 10. Comparative analysis of different frequency harmonic current amplitude

In Fig.10, the 3rd harmonic is the main harmonic component in the simulation curve and the actual curve, so the 3rd harmonic needs to be the key suppression component.

\section{Conclusion}

In this paper, the dynamic model experiment platform of charging pile network distribution network was built, and the parameters of power supply network, charging pile and simulated load were designed.

The research conclusions are as follows:(1) With the increase of CP number, harmonic current content will decrease correspondingly, and the main component is low frequency odd harmonics; (2)When $16 \mathrm{CP}$ are connected in parallel, the third harmonic is the main component. The waveform of the simulated curve is consistent with that of the measured curve, and the harmonic component is basically consistent. The dynamic model can be used for CP harmonic current analysis, which provides an effective basis for domestic and foreign research institutes to carry out relevant tests.

\section{References}

[1] Serpa L A, Ponnaluri S. A Modified Direct Power Control Strategy Allowing the Connection of Threephase Inverters to the Grid Through LCL Filters $[\mathrm{J}]$. IEEE Transactions on Industry Applications, 2007, 43 (5) : 1388-1400.

[2] B. Jiang, X. Z. Dong, S. X. Shi. Experimental Research on Typical Method of Single Phase Grounding Fault Line Selection in Distribution Network [J]. Electric power automation equipment, 2015,35(11):6774.

[3] F. G. Zhang, T. Wu, G. S. Yi, et al. Research on dynamic model of distribution network based on fault location test [J]. Water and power energy science,2018,36(09):167-171.

[4] X. F. Tao. Design and Implementation of Automatic Test Platform for ELECTRIC Vehicle DC Charging Pile [D]. University of Electronic Science and Technology of China,2018.

[5] W. Chen, J. Zhou, Z. Xu, et al. Electric measurement \& instrumentation,2019,56(06):26-31+49.

[6] J. Zhou, G. Y. Ren, C. Wei, et al. Electric power system protection and control,2017,45(05):18-25.

[7] B. B. Zhong. Harmonic Analysis of Electric Vehicle Charging Station [D]. Huazhong University of Science and Technology, 2015.

[8] W. S. Song, X. Y. FENG. An Internal Relation between Single-phase three-level SVPWM modulation and Carrier SPWM [J]. Transactions of China Electrotechnical Society, 2012, 27(6): 131-138. 